\title{
On Leadership Development in the National Health Service Scotland
}

\author{
T. S. Durrani and S. M. Forbes \\ Faculty of Engineering \\ University of Strathclyde \\ Glasgow G1 1XW, Scotland UK \\ durrani@strath.ac.uk : s.forbes@eee.strath.ac.uk
}

\begin{abstract}
The National Health Service (NHS), which delivers healthcare to all in the $\mathrm{UK}$, is undergoing profound change, from the role of the primary-care providers, the general practitioners, to the specialist hospital managers. There is a drive that ranges from greater care in the community to more effective use of highly specialist resources in hospitals. This is linked to the restructuring of the management in the NHS, requiring a new breed of leaders to achieve organisational change, and to attain development and growth. To develop the new cadre of managers, an exciting Programme is being established for Leadership and Organisational Development. This paper reflects on the background to the change in the NHS, and provides an account of the Leadership Development Programme that is being implemented in the NHS Scotland.
\end{abstract}

\section{INTRODUCTION}

The National Health Service (NHS) in the UK is now fifty-eight years old. It was established in 1948, with the abiding principal of providing healthcare for all UK citizens, based on the need and not the ability to pay. Funding for the Service comes from the UK taxpayer, and is managed by the Government Department of Health, which sets overall policy on health issues.

In terms of local delivery of services, the NHS is organised through a series of regional entities, called Health Boards. The NHS has undergone a fundamental change in recent years, and a current review (the Kerr Report: 'Building a Health Service Fit for the Future' [1]), has made major recommendations that will impact on the way the service is managed, particularly in Scotland. The dominant issues, highlighted by public and healthcare professionals, include the need for:

- Maintaining high quality services locally.

- Improving waiting times.

- Supporting Scotland's remote and rural communities.

- Empowering clinical staff to meet the challenge of reforming the Health Service.

- Using new technology to improve the standard of care.

- Reducing the health gap between rich and poor.

- Ensuring that NHS delivers value for money.
The impact of the review is far-reaching, both in terms of organisational development and resource management, aimed at ensuring increased productivity and better management of services on behalf of patients. This paper highlights issues being faced by senior management in delivering the type of service envisaged in the Kerr Report [1] and, in particular, addresses a new initiative being taken to reinforce and cultivate a cadre of leaders who would progress the modernisation agenda.

\section{New Structure For The NHS}

In recent years, professional administrators, who have overseen the delivery of services, management of resources, and the utilisation of clinical personnel, have managed the NHS hospitals, while local healthcare in the UK has always been provided by General Practitioners (GPs), spread geographically, and working quasi-independently from the large hospitals; though the GPs have the facility to refer patients to the large hospitals for serious attention and support, and clinical care.

A national framework has recently been proposed [2] for service change, to develop sustainable specialist services along with more local services delivered in community settings. The objective is to reinforce and enrich 'patient pathways' from community care and GP oversight, through to specialised care, with to the use of information technology that links all aspects of clinical care, covering primary and secondary networks of rural hospitals supported by the major teaching hospitals, and through careful planning for specialised services such as neurosurgery, etc.

To achieve this new structure, there is a need for the development of a new cadre of effective managers, equally at home with technology as with government policy, with a clear understanding of community care principles and the optimal utilisation of resources. In this development process primacy is being given to clinical directors - who are clinicians (professional medics) taking up management responsibilities. To this effect, a major programme is being launched between the University of Strathclyde and the NHS Greater Glasgow to develop the Leaders for tomorrow for the NHS 


\section{THE KERR REPORT}

Professor Kerr's Report [1] outlines a twenty-year plan for the NHS in Scotland. Rather than focusing on one single part of the health service, it considers the NHS as a whole, and provides a unique perspective on where and how services should be delivered to patients in Scotland.

The Report proposes reforms and roles for three specific healthcare areas that embody the kernel of the National Health Service:

- General practice and primary care.

- Secondary care and hospital services.

- Staff recruitment and retention.

\section{A. General Practice and Primary Care}

The Report places emphasis on the care that is provided to patients in their local communities, primarily in general practice. General Practitioners (GPs), are the local doctors operating from a local medical centre, which is the first port of call for all patients. GPs play a key role in ill health prevention, chronic disease management and are responsible for the healthcare of every one of their patients. GPs are in effect the 'gatekeepers' to other parts of the NHS, and are responsible for referring patients to general hospitals for more specialist treatment. There is an increasing emphasis on broadening the remit of general practice to provide more specialist care in the community. The Report sets out key challenges for general practice, emphasising the need for anticipatory care and focusing on preventing hospital admissions.

Professor Kerr clearly identifies an important role for Community Health Partnerships (CHP) in his proposed reforms. Integrating primary and secondary care will be of vital importance to the success of CHPs. The NHS in Scotland needs to ensure that it has the flexibility and capability to provide such a patient-centred service for a whole range of conditions.

\section{B. Secondary Care and Hospital Services}

Professor Kerr's proposals include plans to separate elective and emergency care, specifically through the introduction of diagnostic and treatment centres. A benefit of this approach would be to reduce the number of operations that are cancelled because of pressures elsewhere in the system. It is, however, important that more effective services are provided without increasing bureaucracy, and minimising barriers to the access of services.

The objectives of the recommendations are to improve regional planning in terms of the service redesign that is required to make the NHS in Scotland more efficient. In this context, while some complex procedures can only be carried out in centralised units, there are many procedures that can be managed locally, with different approaches required for rural and urban communities.

\section{Staff Recruitment and Retention}

Recruitment of doctors is a serious problem particularly in the rural parts of Scotland. This is exacerbated by the need to reduce doctors' working hours and the changing demography of the medical profession. There is general support for the extension of the roles of other health professionals, who have valuable skills that can contribute to the provision of service. Nevertheless, doctors are highly qualified and skilled professionals, and should retain the core duties of diagnosing, treating and managing patients. With a shift in emphasis away from hospital-based care, there is a need for an increase in the number of GPs and attendant training places in Scotland to ensure future GP workforce requirements are met. In order to facilitate this, medical students and doctors in training should have more exposure to general practice to encourage them into this branch of the profession.

An important element of the retention issue is the need for the development of effective leaders who would oversee the change, and establish vehicles for the delivery of a more effective and efficient National Health Service. To this end an innovative Programme is being developed to produce the 'Leaders for Tomorrow', who would manage the revitalised and restructured Health Service. The Programme is being developed in partnership with the University of Strathclyde in Glasgow, in 2006, details of which are given below.

Fig. 1 gives the profile of the current staffing complement at NHS Scotland. At 30th September 2005 there were 153,996. It may be surmised that with the significant spread in the staff categories, there is a need to build leadership potential in all cadres of staff [3]

\section{The Leadership DeVelopment Programme FOR THE NHS IN SCOTLAND}

The 2005 Scottish Executive paper 'Delivery Through Leadership: NHS Leadership Development Framework' [2] identified three key priorities:

- Building overall leadership capacity and growing new and emerging leaders.

- Developing leadership capability to meet the critical aspects of the NHS Scotland change agenda and to deliver real results.

- Supporting career development for leaders.

The Framework approach to leadership development is distinct and different, in that it is aimed at transformational change rather than management development, where the latter is concerned with ensuring improvements in transactional and operational processes and problem solving. 
The aspirations of the Framework are "to permeate leadership qualities in each ward team, community team, functional team, to make a difference". Nevertheless, there is recognition that senior managers are seen as role models, hence, the focus in the initial stages of developing the senior managers and senior clinical leaders. The expectation is that clinical leaders will drive service improvement and effective management teams to deliver excellent patient care.

The leadership development initiative is embedded within the context of a major change agenda in NHS Scotland and NHS Greater Glasgow. The drivers for this change include: the need to develop and implement service strategies to improve healthcare delivery; the requirement to address rising public expectations for a first-rate quality of service; the necessity to optimise the use of resources, and their concomitant impact on health improvement; the move to change the focus from service and performance focus of institutions to that of networks that form a "continuum of care' and form healthcare to health. Another important aspect of the Framework is to embed healthcare in a 'partnership' comprising a wide sector of public service agencies, integrating a range of services already being provided, and thus giving form to the concept of "care in the community'.

The linkages between the different elements of the Framework are identified in Figure 1, where the interactions between the three key priorities are clearly identified, and the constituent elements are illustrated. It may be seen that the Framework lends strategic coherence by providing focus on the requirements of the NHS, the associated teams and individuals. The need to develop clinical leadership is seen as of primary importance, blending with the development of strategic teams, and taking into account the need for succession planning. To build on this, and to enhance Leadership Capacity, there is a need to enhance the leadership potential of Clinical Health Partnerships, Clinical Executives, and management resources in the Clinical/Care Networks. Intimately related to the two priorities is the requirement to develop strategies for career enhancement and development, providing ladders for top leaders, as well as careful management of the careers of the local staff, and investing in a skills bank.

To meet these priorities and, in particular, to address the challenges of effective leadership and management in NHS Greater Glasgow, as identified in 'Partnership for Care reforming the NHS in Glasgow' [2], a pan-Glasgow Leadership Programme is proposed. Initially the Programme provides a focus on the newly appointed clinical directors and Community Health Partnership associate medical directors, with a development programme for other staff to be taken forward as a follow on.
The Programme offers an understanding of the changing environment of the Health Service, and aims to develop an appreciation of the new leadership roles needed to maintain and grow the innovative spirit in NHS organisations through the delivery of improved healthcare services. The first priority has been addressed through the identification of leadership competencies and the subsequent selection process. The proposed Programme is directed towards meeting the other two priorities.

The Programme recognises the prior competencies of the participants. However, the aim is to support participants to become excellent clinical leaders by improving performance. By the end of the Programme, participants are expected to be effective leaders, with a new skill set which will enable them to provide competent and innovative directions. The overarching aim is to consider, and to deliver, changes in clinical service delivery for the benefit of patients.

\section{A. Key Objectives}

The objectives of the Programme are:

- To prepare and support participants for senior leadership and management roles.

- To enhance participants existing knowledge and understanding of issues facing the NHS.

- To build a cohort of leaders through a shared learning experience as a contribution towards organisational development.

- To enhance strategic thinking and understanding of strategic approaches to change.

- To provide a broader perspective on leading and managing complex services.

- To establish a team of clinical leaders who will work well with general managers to carry forward the drive towards innovation and improvement in the health service.

\section{B. Learning Outcomes}

Through a series of Workshops and a Group Assignment the participants are expected to develop:

- An awareness of leadership traits and strategic management processes to provide guidance and organisational change.

- Knowledge of environmental and cultural influences on NHS Scotland in its role as a local, regional and national entity.

- A network of leaders who have an appreciation of governance and management challenges facing the NHS.

- Appreciation of internal and external environments within which NHS Scotland operates.

- Insight into the dynamic and reinforcing relationships between clinical governance, effective management, and service delivery.

- Awareness of effective multidisciplinary team-working though an application in the participant's own teams. 


\section{PROPOSED APPROACH}

The approach taken to the learning process entails:

- Presentations by Health Service leaders and other experts in their fields at residential workshops.

- Small group discussions.

- Group problem-solving exercises.

- Networking opportunities for delegates.

- Reference material, course notes, and copies of slide presentations.

- Group Assignments.

- Mentoring by local supervisors.

- Final presentation of assignments to senior executives of NHS Greater Glasgow.

The Programme comprises three residential two-day Workshops, a Group Assignment, plus a final session where the results of the Assignments are presented to senior management in the NHS. The Group Assignment is an important aspect of the developmental and learning process. The topics for the Assignments are provided by senior NHS Management, and are aimed at addressing critical and realistic issues currently facing the NHS in Glasgow. In some cases the Assignments are directly linked to the key themes which are the focus of the new organisation, such as: addressing health improvement and tackling inequalities; taking responsibility for quality of service delivered to patients; or, driving integration of community, health and social services to improve the experience of patients. The participants are organised in groups, which reflect a mix of backgrounds and experiences, and some gender balance. The groups conduct studies off-line between the workshops, and the results from the Assignments are presented to Board members of NHS Greater Glasgow.

To assist with the cohesion of each group, a mentor/supervisor is identified who oversee the development of the Assignment and provide assistance and support. In particular, paired non-executive Directors of NHS Greater Glasgow, who can be lay members, or practicing medics are ideally placed to act as group mentors/supervisors. There is some merit in matching nonexecutives leading on specific topics (e.g. governance, clinical health practitioners) with participants who may be most influential in the relevant area.

\section{SPEAKERS}

While specialist speakers are provided to cover specific management subjects, such as leadership traits or strategic management; in order to ensure credibility and to expose the participants to authorities in the health-care field, invited keynote speakers from the Health Service will make specific presentations on topics of direct relevance to the NHS.

\section{PRIOR INFORMATION}

The following information is required prior to the start of the Programme:

- Background on participants (e.g. self-written short biographies with professional and non-professional information to provide a values base and to support networking).

- Response to a questionnaire focussing on aspirations and motivations of individuals.

- Suggestions from participants on local leadership/ management issues for possible discussion/inclusion in the Programme.

- Proposal for Group Assignments by senior staff.

\section{LOCATION, NUMBERS AND TIMESCALES}

The Workshops are held at a residential location, aimed at assisting with ease of learning, networking, discussion and debate in an informal setting. It is important that the Programme is delivered in a setting, which takes delegates out of their usual work environment. To ensure that the participants benefit from small group interaction and individual attention, between twelve and fifteen participants per workshop are chosen. It will be important to engender a good mix of candidates by aiming a reasonable balance of gender, experiences, responsibilities and professional backgrounds.

An important element of the Programme is a formal evaluation to assess the impact of the Programme, the effect and value of the learning process and behaviour change.

\section{Final COMMENTS}

The National Health Service in the UK in general, and Scotland in particular, is undergoing a significant change, and to meet the requirements of the restructured Health Service, both at primary care and secondary care level, as well as the need for engagement with the community, a clear requirement has been identified for organisational development across the board. To this effect a new cadre of individuals are being developed who would manage the change agenda, and lead the NHS to deliver the service befitting the twenty-first century in the context of vision of the founding fathers - that

of a universal Service for all based on clinical need and not the ability to pay.

This paper presents a Programme for the development of clinical individuals who would deliver this vision.

\section{REFERENCES}

[1] A. Kerr, 'Buildng a Health Service fit for the future' http://www.scotland.gov.uk/Publications/2005/05/23141307/13135

[2] Scottish ExecutivePublications, http://www.scotland.gov.uk/Publications/2005/06/28112744/27466

[3] Scottish Health Statistics, http:/www.isdscotland.org/. 
Fig 1

\section{Profile of Headcount staff as at}

30 September 2005
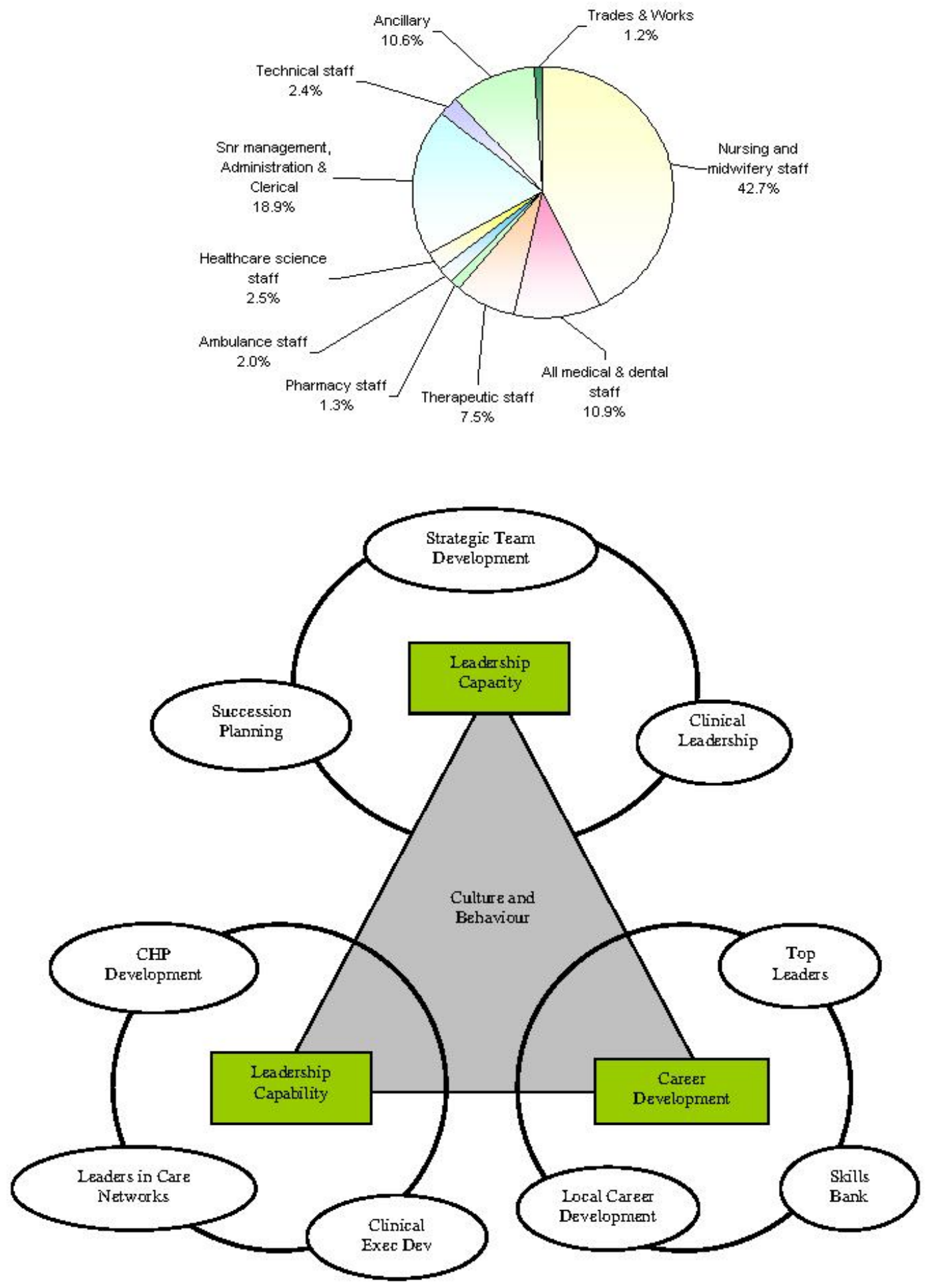

Fig 2. Leadership Development Framework [2] 